\title{
Big double bubble trouble: in vivo real time demonstration of 'mixed-type bubble' and its consequent effects during deep anterior lamellar keratoplasty
}

\author{
Harathy Selvan ${ }^{1} \cdot$ Shikha Yadav $^{1} \cdot$ Radhika Tandon $^{1}$ \\ Received: 2 January 2018 / Accepted: 11 January 2018 / Published online: 16 February 2018 \\ (c) The Royal College of Ophthalmologists 2018
}

The 'big-bubble' deep anterior lamellar keratoplasty (DALK) technique paved the way to redefine corneal anatomy by discovery of a new 'pre-Descemet/Dua's layer'. The study of evolution and morphology of these bubbles led them to be classified into three types [1]. Type-1 bubble is a domeshaped elevation that forms centrally, spreading centrifugally between the stroma and pre-Descemet layer, while the type- 2 is a larger, clear-edged thin-walled bubble, forming peripherally and expanding centripetally between the pre-Descemet layer and the Descemet membrane (DM). The third uncommon type is the 'mixed bubble' where usually there is a primary type-1 bubble along with a secondary type- 2 bubble, which may even be hidden under the semi-opaque former one $[1,2]$. This identification is important to foresee the specific complications such as the higher tendency of bursting of the type- 2 component. Though studied extensively, yet they have been rarely documented in vivo [1-3].

We demonstrate a mixed bubble during DALK, by in vivo intra-operative video photography, clearly displaying the inception of both the bubbles (Supplemental Video file and Fig. 1a). Microscope-integrated optical coherence tomography (MiOCT) was used to capture this rare scenario, where it revealed two taut layers beneath the stroma, the pre-Descemet layer and DM, respectively, separated by tense intracompartmental air (Fig. 1b). The type-1 bubble was then deflated and host stromal dissection continued to bare the preDescemet layer (Fig. 2a). Despite leaving the type-2 bubble

Electronic supplementary material The online version of this article (https://doi.org/10.1038/s41433-018-0038-y) contains supplementary material, which is available to authorized users.

Radhika Tandon

radhika_tan@yahoo.com

1 Cornea and Refractive Services, Dr Rajendra Prasad Centre for Ophthalmic Sciences, All India Institute of Medical Sciences, New Delhi 110029, India intact, extensive DM detachment (DMD) was visible under the MiOCT system during the course of surgery (Fig. 2b). Intracameral air injection at the end led to successful apposition of the DM to the pre-Descemet layer.

The most common and preferred type of big-bubble for DALK is the type-1, owing to the additional strength provided by the retained host pre-Descemet layer. A mixed bubble also confers the same advantage, but additionally provides the risk of DMD. To circumvent this, AlTaan et al. [3] suggested an intentional puncture of the pre-Descemet layer to release the type-2 air pocket off the mixed bubble. However, postoperative DMD was still noticeable when a patient incurred accidental intra-operative perforation during a mixed-bubble DALK [4]. Spontaneous resorption of the type-2 air pocket in the post-operative period has also been shown [5]. However, both these practices could not evade post-operative DMD because the mixed-type bubble intra-operatively creates an additional deeper enclosed space between the pre-Descemet layer and DM, and as both these layers are left intact, this space may potentially enlarge or be prevented from spontaneous resolution by the persistence of air or seepage of fluid. On the contrary, in pure type- 1 or type- 2 bubbles, when formed singly, the space created is deroofed during dissection.

Considering this high propensity of DMD and lack of consensus on the management of the deeper type- 2 component of a mixed bubble, the surgeon should be alert and aware to the clinical signs of mixed bubble forming and the possible need for intracameral air injection at the end of surgery to reverse the DMD by the strategy of internal tamponade, evading unnecessary post-operative patient morbidity. MiOCT provides an upper hand in the identification and management of such situations.

\section{Disclaimer}

All authors have read and approved the final version of this manuscript. The requirements for authorship have been met 

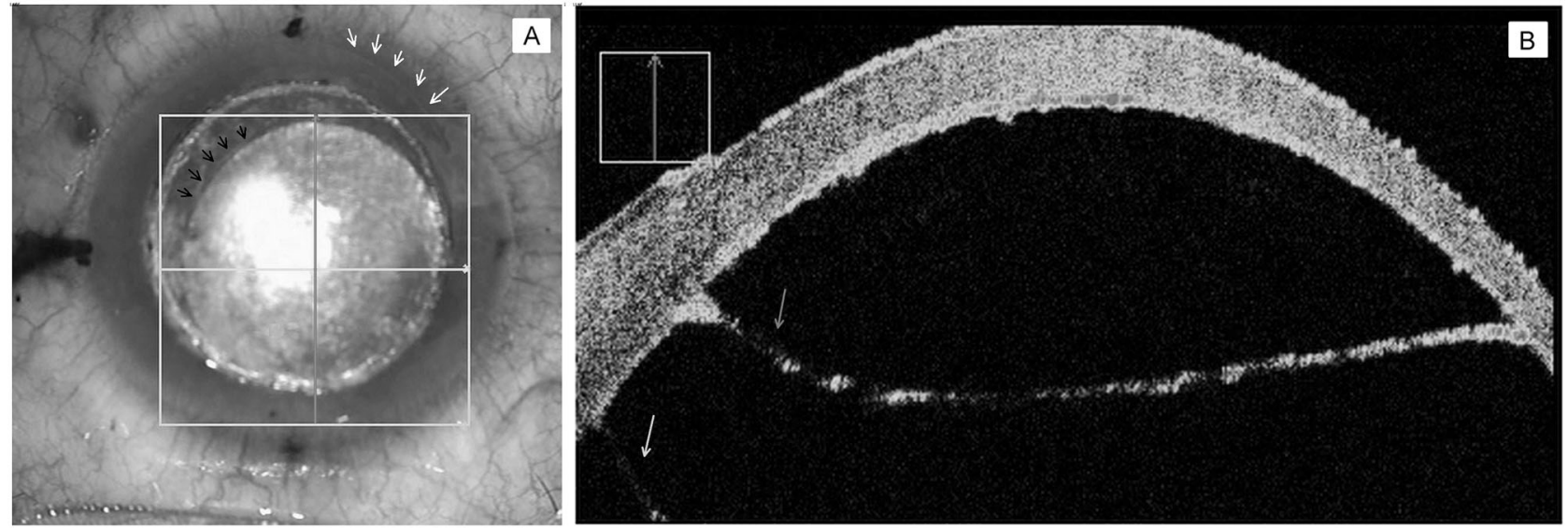

Fig. 1 a Intra-operative photograph of in vivo mixed bubble where the black arrows highlight the edges of type-1 bubble within the edges of trephination and white arrows delineate the edges of larger type- 2 bubble extending close to the limbus. b Microscope-integrated optical coherence tomography (MiOCT) image of Fig. 1a depicting the cleavage plane of type- 1 bubble between the stroma and pre-Descemet's layer (red arrow), and of type-2 bubble between the latter and Descemet membrane (yellow arrow) of the host corneal tissue. (The entire bubble could not be captured due to the limited depth of focus of $\mathrm{MiOCT})$. (color figure online)
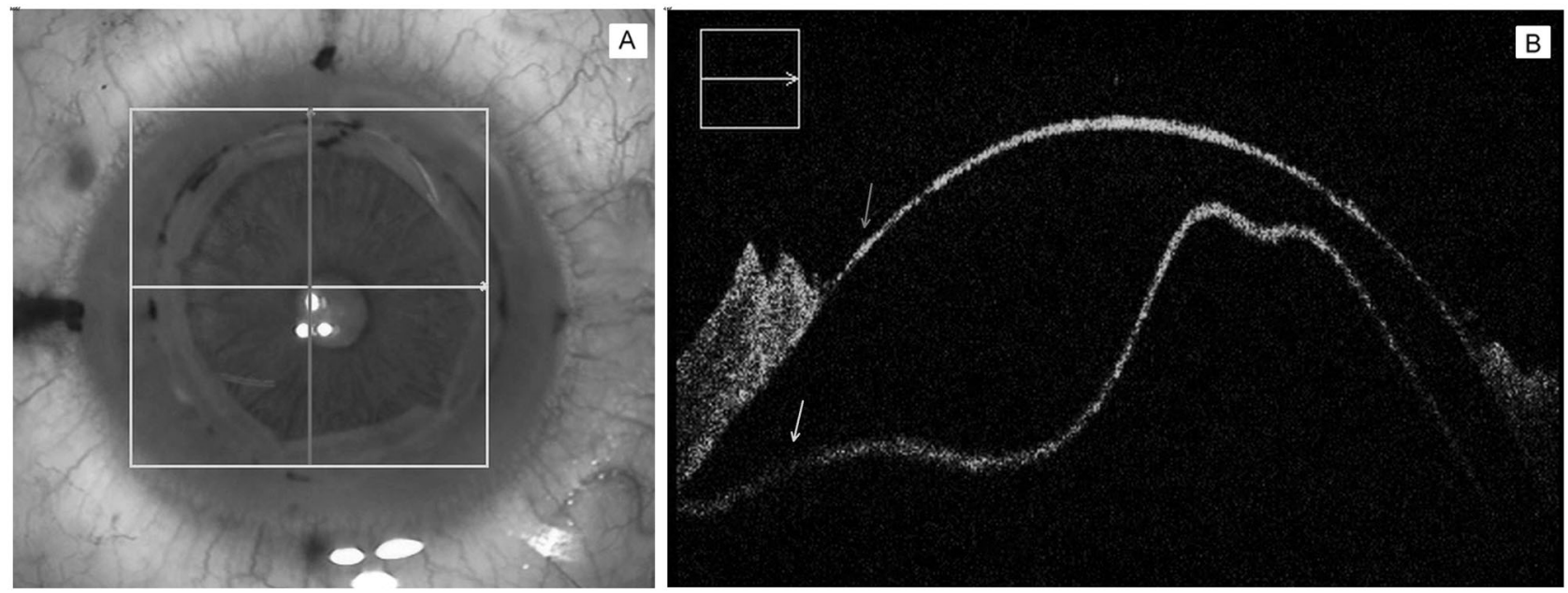

Fig. 2 a Intra-operative photograph of host bed, baring the pre-Descemet layer. b MiOCT image of Fig. 2a showing the pre-Descemet layer (red arrow) and the detached, Descemet membrane (yellow arrow). (color figure online)

by each author, and all of us believe that this manuscript represents our honest work.

\section{Compliance with ethical standards}

Conflict of interest The authors declare that they have no conflict of interest.

\section{References}

1. Dua HS, Faraj LA, Said DG, Gray T, Lowe J. Human corneal anatomy redefined: a novel pre-Descemet's layer (Dua's layer). Ophthalmology. 2013;120:1778-85.
2. Goweida MBB. Intraoperative review of different bubble types formed during pneumodissection (big-bubble) deep anterior lamellar keratoplasty. Cornea. 2015;34:621-4.

3. AlTaan SL, Termote K, Elalfy MS, Hogan E, Werkmeister R, Schmetterer L, et al. Optical coherence tomography characteristics of different types of big bubbles seen in deep anterior lamellar keratoplasty by the big bubble technique. Eye. 2016;30:1509-16.

4. Fuest M, Mehta JS. Descemet membrane splitting following deep anterior lamellar keratoplasty. JAMA Ophthalmol. 2017;135: e170656

5. Wise S, Dubord P, Yeung SN. Double bubble with the big-bubble technique during deep anterior lamellar keratoplasty. Int Ophthalmol. 2017. Apr 28. https://doi.org/10.1007/s10792-017-0540-4. [Epub ahead of print] 